\title{
A multi-mode area-efficient SCL polar decoder
}

\author{
Chenrong Xiong, Jun Lin, Student member, IEEE and Zhiyuan Yan, Senior member, IEEE
}

\begin{abstract}
Polar codes are of great interest since they are the first provably capacity-achieving forward error correction codes. To improve throughput and to reduce decoding latency of polar decoders, maximum likelihood (ML) decoding units are used by successive cancellation list (SCL) decoders as well as successive cancellation (SC) decoders. This paper proposes an approximate ML (AML) decoding unit for SCL decoders first. In particular, we investigate the distribution of frozen bits of polar codes designed for both the binary erasure and additive white Gaussian noise channels, and take advantage of the distribution to reduce the complexity of the AML decoding unit, improving the area efficiency of SCL decoders. Furthermore, a multi-mode SCL decoder with variable list sizes and parallelism is proposed. If high throughput or small latency is required, the decoder decodes multiple received codewords in parallel with a small list size. However, if error performance is of higher priority, the multi-mode decoder switches to a serial mode with a bigger list size. Therefore, the multi-mode SCL decoder provides a flexible tradeoff between latency, throughput and error performance, and adapts to different throughput and latency requirements at the expense of small overhead. Hardware implementation and synthesis results show that our polar decoders not only have a better area efficiency but also easily adapt to different communication channels and applications.
\end{abstract}

Index Terms-Error control codes, polar codes, successive cancellation list decoding, ML decoding, multi-mode decoding, parallel decoding

\section{INTRODUCTION}

Polar codes [1], a breakthrough in coding theory, have attracted lots of research interest since they achieve the symmetric capacity of memoryless channels with both binary input [1] and nonbinary input [2]. A lot of effort has been made to improve the error performance of polar codes with short or moderate lengths $[3]-[8]$, and to improve the hardware area efficiency of polar decoders [9]-[20].

Maximum likelihood (ML) decoding algorithms - the sphere decoding algorithm [6], stack sphere decoding algorithm [7] and a Viterbi algorithm [8] — can be used to decode polar codes, but their complexity can be prohibitively high. Compared with ML decoding algorithms, the successive cancellation (SC) decoding algorithm [1] has a lower complexity at the cost of sub-optimal performance. To improve the performance of the SC algorithm, the SC list (SCL) decoding algorithm [21] and the CRC-aided SCL (CA-SCL) algorithm [3], [5] were proposed. A key drawback of the SC, SCL and CA-SCL algorithms is their long decoding latency and low decoding throughput, as these algorithms deal with only one bit at a time: the SC algorithm makes hard bit decisions only one bit at a time; in the SCL and CA-SCL algorithms, the path expansion is with respect to only one bit.

To reduce decoding latency and improve throughput of an SC polar decoder, several algorithms [13], [15], [22], [23] were proposed to deal with several bits at a time instead of only one bit by using ML decoding units, which calculate symbolwise channel transition probabilities and make hard decisions for several bits at a time. Based on the SC algorithm, the parallel SC [22], hybrid ML-SC [23], ML simplified SC (MLSSC) [15] and fast ML-SSC [13] algorithms were proposed. The basic difference of ML decoding units between these four algorithms is that hybrid ML-SC [23] and fast ML-SSC [13] take advantage of the distribution of frozen bits to reduce complexity, but neither parallel SC [22] nor ML-SSC [15] algorithms do so.

ML decoding units in [19], [20], [24]- 27] are also used to improve throughput of SCL-based decoders and to reduce decoding latency. Instead of making hard decisions in SCbased algorithms, an ML decoding unit for SCL-based algorithms calculates symbol-wise channel transition probabilities and performs path expansion and pruning. None of these SCLbased algorithms takes advantage of the distribution of frozen bits to reduce complexity of ML decoding units. Therefore, ML decoding units in these SCL-based algorithms have high complexities. For example, when the list size is four and the symbol size is eight, the ML decoding unit accounts for $27 \%$ of the overall decoder area in [25]. In [27], when the code length is 1024 , the area of an ML decoding unit takes up as much as $62 \%$ of the overall decoder area.

In this paper, we first propose a low-complexity approximate ML (AML) decoding unit by utilizing the distribution of frozen bits of polar codes and then propose a multi-mode SCL (MM-SCL) polar decoder to support variable throughput and latency. Our main contributions are:

- The divide-and-conquer method in [23] is applied in the probability domain to simplify the ML unit for SCbased algorithms. By extending this idea, a divide-andconquer AML decoding unit for SCL-based algorithms is proposed by considering the distribution of frozen bits. Its computational complexity is greatly smaller than those of existing ML decoding units for SCL-based algorithms. When an appropriate design parameter for the divide-andconquer AML decoding unit is selected, the SCL decoder has negligible performance loss.

- The distribution of frozen bits of polar codes is analyzed from the viewpoint of code construction. We show that there are only a small number of frozen-location patterns for polar codes constructed by a method proposed by Arıkan in [28] and a method in [29].

- Since only a small number of frozen-location patterns exist in polar codes, the divide-and-conquer AML decoding unit for SCL-based algorithms is simplified further. A low-complexity hardware implementation for the simplified divide-and-conquer AML decoding unit, the LC-AML decoding unit, is proposed. Synthesis results show that by taking advantage of a small number of 
frozen-location patterns, our CA-SCL decoder with the LC-AML unit has a better area efficiency than existing SCL decoders, while working for all channel conditions.

- An MM-SCL polar decoder is also proposed. This decoder supports SCL algorithms with different list sizes and parallelism. When a high throughput or small latency is needed, the MM-SCL decoder decodes multiple received codewords in parallel with a small list size. If a good performance is required, the MM-SCL decoder switches to a mode with a greater list size to decode only one receive codeword. Therefore, the MM-SCL polar decoder provides a flexible tradeoff between latency, throughput and performance, and consequently adapts to different throughput and latency requirements at the expense of small overhead.

Our proposed divide-and-conquer AML decoding unit for SCL-based algorithm is an extension of the method for SCbased algorithm in [23]. However, by investigating the distribution of frozen bits of polar codes, we reduce the complexity of the ML decoding unit further. Existing ML decoding units for SCL decoders [19], [24]-[27] perform list pruning function after all the symbol-wise channel transition probabilities are calculated, whereas the LC-AML decoding unit sorts intermediate calculation results generated by the recursive channel combination method [19], and consequently reduces the number of symbol-wise channel transition probabilities dealt with by the list pruning function. Hence, the proposed LC-AML decoding unit has a much smaller complexity. The performance degradation due to the proposed LC-AML is the same as that in [19], [25]. Although the ML decoding unit in [24] has no performance degradation, its complexity grows quickly as the list size and symbol size increase. The performance degradation of the ML decoding unit in [26], [27] depends on its design parameters.

Many applications, such as modern wireless or wireline communication system, require variable data rate transmission and have stringent latency requirements. As a potential candidate of FEC technique for future communication systems, a polar decoder supporting variable data rate and variable decoding latency is desired. Unfortunately, existing polar decoders provide only fixed latency and throughput (data rate). To the best of our knowledge, the proposed MM-SCL decoder is the first polar decoder with variable throughput and decoding latency given a polar code.

The rest of this paper is organized as follows. In Section II polar codes as well as construction methods for polar codes and existing ML decoding units are reviewed. In Section III. first, the divide-and-conquer method is applied in the probability domain for the ML unit of SC-based algorithms. Then, the divide-and-conquer AML decoding unit for SCL-based algorithms is proposed, and its computational complexity is also analyzed. In Section IV frozen-location patterns for polar codes are investigated. In Section $\mathrm{V}$, a hardware design of the LC-AML unit is proposed, and an area-efficient CA-SCL decoder with the LC-AML unit is implemented as well. The hardware implementation and synthesis results for the areaefficient SCL decoder are also presented in this section. In Section VI] the MM-SCL decoder, its hardware implementation and synthesis results are presented. Finally, some conclusions are provided in Section VII

\section{PREliminaries}

\section{A. Notations}

Suppose $\mathbf{u}$ represents a binary vector $\left(u_{1}, u_{2}, \cdots, u_{N}\right) . u_{a}^{b}$ denotes a binary subvector $\left(u_{a}, u_{a+1}, \cdots, u_{b-1}, u_{b}\right)$ of $\mathbf{u}$ for $1 \leq a, b \leq N$; if $a>b, u_{a}^{b}$ is regarded as void. $u_{a, o}^{b}$ and $u_{a, e}^{b}$ represent the subvectors of $u_{a}^{b}$ with odd and even indices, respectively. For an index set $\mathcal{A} \subseteq \mathcal{I}=\{1,2, \cdots, N\}$, its complement in $\mathcal{I}$ is denoted as $\mathcal{A}^{c}$. The subvector of $\mathbf{u}=u_{1}^{N}$ restricted to $\mathcal{A}$ is represented by $\mathbf{u}_{\mathcal{A}}=\left(u_{i}: 0<i \leq N, i \in\right.$ $\mathcal{A})$.

\section{B. Polar codes}

For an $(N, K)$ polar code, the code length $N$ is a power of two, i.e., $N=2^{n}$ for $n>0$. The data bit sequence, represented by $\mathbf{u}=u_{1}^{N}$, is divided into two parts: a $K$ element part $\mathbf{u}_{\mathcal{A}}$ which carries information bits, and $\mathbf{u}_{\mathcal{A}^{c}}$ whose elements (called frozen bits) are set to zero. The corresponding encoded bit sequence $\mathbf{x}=x_{1}^{N}$ is generated by $\mathbf{x}=\mathbf{u} B_{N} F^{\otimes n}$, where $B_{N}$ is the $N \times N$ bit-reversal permutation matrix, $F=\left[\begin{array}{ll}1 & 0 \\ 1 & 1\end{array}\right]$, and $F^{\otimes n}$ is the $n$-th Kronecker power of $F[1]$.

\section{Construction Methods of Polar Codes}

An essential problem for constructing a polar code is to determine the locations of frozen bits (elements of $\mathcal{A}^{c}$ ). For the BEC with an erasure probability $\epsilon(0<\epsilon<1)$, assuming $z_{0,1}=\epsilon$, the following recursions [28] are used to construct an $(N, K)$ polar code, where $N=2^{n}$ and $0<K<N$ :

$$
\begin{gathered}
z_{i, 2 j-1}=2 z_{i-1, j}-z_{i-1, j}^{2}, \\
z_{i, 2 j}=z_{i-1, j}^{2}
\end{gathered}
$$

where $1 \leq i \leq n$. Then $\mathcal{A}^{c}$ is chosen such that $\sum_{j \in \mathcal{A}^{c}} z_{n, j}$ is maximal and $\left|\mathcal{A}^{c}\right|=N-K$.

For the AWGN channel and a given initial value of $z_{0,1}$, which is determined by a desired signal-to-noise ratio (SNR), the following recursive method [29] based on Gaussian approximation is used for $1 \leq i \leq n$ :

$$
\begin{gathered}
z_{i, 2 j-1}=\tau^{-1}\left(1-\left(1-\tau\left(z_{i-1, j}\right)\right)^{2}\right) \\
z_{i, 2 j}=2 z_{i-1, j}
\end{gathered}
$$

where

$$
\tau(x)= \begin{cases}e^{-0.4527 x^{0.86}+0.0218}, & 0<x<10 \\ \sqrt{\frac{\pi}{x}} e^{-\frac{x}{4}}\left(1-\frac{10}{7 x}\right) & x \geq 10 .\end{cases}
$$

In this case, $\mathcal{A}^{c}$ is chosen such that $\sum_{j \in \mathcal{A}^{c}} z_{n, j}$ is minimal and $\left|\mathcal{A}^{c}\right|=N-K$. 


\section{Existing ML Decoding Units for Polar Decoders}

When $\mathbf{x}=\mathbf{u} B_{N} F^{\otimes n}$ is transmitted, suppose the received word is $\mathbf{y}=y_{1}^{N}$ and the symbol size is $M=2^{m}$. A symboldecision [19] ML decoding unit first calculates symbol-wise channel transition probabilities, $\operatorname{Pr}\left(\mathbf{y}, \hat{u}_{1}^{j M} \mid u_{j M+1}^{j M+M}\right)(0 \leq j<$ $\left.\frac{N}{M}\right)$, then makes a symbol-wise ML decision for SC-based decoders or chooses the $L$ most reliable paths for SCL-based decoders. Here, $\hat{u}_{1}^{j M}$ is the previously estimated bits.

There are three methods to calculate the symbol-wise channel transition probabilities, and all of them do not take advantage of the distribution of frozen bits. The first [15], [22], [24], [26] is based on an $M$-element product of bitwise channel transition probabilities, called directing mapping method (DMM):

$$
\begin{aligned}
& \operatorname{Pr}\left(\mathbf{y}, \hat{u}_{1}^{i M-M} \mid u_{i M-M+1}^{i M}\right)= \\
& \quad \prod_{j=0}^{M-1} \operatorname{Pr}\left(y_{j \frac{N}{M}+1}^{(j+1) \frac{N}{M}}, \hat{w}_{1+j \frac{N}{M}}^{(i-1)+j \frac{N}{M}} \mid w_{i+j \frac{N}{M}}\right),
\end{aligned}
$$

where $u_{i M-M+1}^{i M}=\left(w_{i}, w_{i+\frac{N}{M}}, \cdots, w_{i+N-\frac{N}{M}}\right) B_{M} F^{\otimes m}$ for $1 \leq i \leq \frac{N}{M}$, and $\hat{w}_{1+j \frac{N}{M}}^{(i-1)+j \frac{N}{M}}$ is the previously estimated bit vector of $w_{1+j \frac{N}{M}}^{(i-1)+j \frac{N}{M}}$.

The second [19], called as the recursive channel combination (RCC) method, is based on a product of symbol-wise channel transition probabilities recursively,

$$
\begin{aligned}
\operatorname{Pr}\left(y_{1}^{2 \Lambda}, \hat{u}_{1}^{i \Phi} \mid u_{i \Phi+1}^{i \Phi+\Phi}\right)= & \operatorname{Pr}\left(y_{1}^{\Lambda}, \hat{u}_{1, o}^{i \Phi} \oplus \hat{u}_{1, e}^{i \Phi} \mid u_{i \Phi+1, o}^{i \Phi+\Phi} \oplus u_{i \Phi+1, e}^{i \Phi+\Phi}\right) \\
& \cdot \operatorname{Pr}\left(y_{\Lambda+1}^{2 \Lambda}, \hat{u}_{1, e}^{i \Phi} \mid u_{i \Phi+1, e}^{i \Phi+\Phi}\right),
\end{aligned}
$$

where $1 \leq \phi \leq m, 0 \leq \lambda<n, \Lambda=2^{\lambda}$, and $\Phi=2^{\phi}$.

The third [27] is a hybrid method by applying the DMM first and then the RCC method, referred to as the DRH method.

Based on the distribution of frozen bits, some data symbols in [13] are considered as some special constituent codes, such as repetition codes and single-parity-check nodes. Different methods were proposed to deal with different constituent codes.

Furthermore, an ML decoding unit in [23] with the divideand-conquer method was proposed for SC algorithms based on an empirical assumption [23]:

Assumption 1. For a well designed polar code, there is no such case that $u_{2 i-1}$ is an information bit and $u_{2 i}$ is a frozen bit, for any $1 \leq i \leq \frac{N}{2}$.

Based on this assumption and the divide-and-conquer method, a simplified ML unit was proposed in [23]. Moreover, a recursive way of the divide-and-conquer method was proposed in [23], but it is not suitable for hardware implementation since it is for a large symbol size, which has a very high complexity for hardware implementation.

How to take advantage of frozen-location patterns to reduce complexity of ML decoding units has been discussed in [13] and [23] for SC-based algorithms, but it has not been investigated yet for SCL-based algorithms.

\section{DIVIDE-AND-Conquer AML DeCoding Unit}

The simplified ML unit in [23] is based on the Euclidean distance since an AWGN channel is assumed. Here, we first apply the divide-and-conquer method in the probability domain and reformulate the ML unit for SC-based algorithms. This simplified ML unit in the probability domain is slightly more general than that in [23], because it is applicable to both AWGN channels and other channels. We then extend the simplified ML unit in the probability domain to SCL-based algorithms.

\section{A. Reformulation of the divide-and-conquer ML unit for $S C$ - based algorithms [23] in the probability domain}

For the ease of discussion, a string vector $\mathbb{S}_{a}^{b}={ }^{\prime} \mathcal{S}_{a} \cdots \mathcal{S}_{b}$ ' (for $1 \leq a \leq b \leq N$ ) is introduced to represent a frozenlocation pattern of symbol $u_{a}^{b}$. If $u_{j}(a \leq j \leq b)$ is an information bit, $S_{j}$ is denoted as ' $\mathcal{D}$ '. Otherwise, $S_{j}$ as ' $\mathcal{F}$ '. Consider a toy example of a four-bit symbol $u_{1}^{4}$. Assuming $u_{1}$ and $u_{3}$ are frozen bits, and $u_{2}$ and $u_{4}$ are information bits. Then the frozen-location pattern $\mathbb{S}_{1}^{4}$ of $u_{1}^{4}$ is ' $\mathcal{F} \mathcal{D F D}$ '. Obviously, for all $M$-bit symbols, there are $2^{M}$ frozen-location patterns.

Based on Assumption 1, $u_{j M+1}^{j M+M}\left(0 \leq j<\frac{N}{M}\right)$ can be divided into $\frac{M}{2}$ pairs, $u_{j M+2 i-1}$ and $u_{j M+2 i}$ for $1 \leq i \leq$ $\frac{M}{2}$. In theory, any pair of $u_{j M+2 i-1}$ and $u_{j M+2 i}$ have four possibilities. ' $\mathcal{F} \mathcal{F}$ ' is trivial. Under Assumption 1, ' $\mathcal{D F}$ ' is not possible. Hence, in [23], only two remaining possibilities are considered: ' $\mathcal{F D}$ ' and ' $\mathcal{D D}$ '. Let $\Omega_{01}^{(j)}$ represent the index set of $i$ that $u_{j M+2 i-1}^{j M+2 i}$ is ' $\mathcal{F D}$ '. $\Omega_{11}^{(j)}$ represents the index set of $i$ that $u_{j M+2 i-1}^{j M+2 i}$ is ' $\mathcal{D D}$ '.

For SC-based algorithms, the maximum of $2^{\left|\Omega_{01}^{(j)}\right|+2\left|\Omega_{11}^{(j)}\right|}$ values of $T\left(u_{j M+1}^{j M+M}\right) \triangleq \operatorname{Pr}\left(\mathbf{y}, \hat{u}_{1}^{j M} \mid u_{j M+1}^{j M+M}\right)$ needs to be found. Based on the RCC method [19], $T\left(u_{j M+1}^{j M+M}\right)=T_{1}\left(v_{\frac{j M}{2}+1}^{\frac{j M+M}{2}}\right) \times T_{2}\left(u_{j M+1, e}^{j M+M}\right)$, where $v_{1}^{\frac{N}{2}} \triangleq u_{1, o}^{N} \oplus u_{1, e}^{N}, \quad T_{1}\left(\frac{\frac{j M^{2}+M}{2}}{\frac{j M}{2}+1}\right) \triangleq \operatorname{Pr}\left(y_{1}^{\frac{N}{2}}, \hat{v}_{1}^{\frac{j M}{2}} \mid v_{\frac{j M}{2}+1}^{\frac{j M+M}{2}}\right)$, and $T_{2}\left(u_{j M+1, e}^{j M+M}\right) \triangleq \operatorname{Pr}\left(y_{\frac{N}{2}+1}^{N}, \hat{u}_{1, e}^{j M} \mid u_{j M+1, e}^{j M+M}\right)$. The possible values of $T\left(u_{j M+1}^{j M+M}\right)$ can be divided into $2^{\left|\Omega_{01}^{(j)}\right|}$ groups based on $\Omega_{01}^{(j)}$, each with $2^{2\left|\Omega_{11}^{(j)}\right|}$ values. In each group, for $i \in \Omega_{11}^{(j)}$, since $v_{\frac{j M}{2}+i}$ and $u_{j M+2 i}$ are independent, $\max (T)=\max \left(T_{1}\right) \max \left(T_{2}\right)$. Then, the maximum of $2^{\left|\Omega_{01}^{(j)}\right|}$ values generated in the previous step is found. Therefore, if $\Omega_{01}^{(j)}=\varnothing$,

$$
\max _{u_{j M+M}^{j M+M}}(T)=\max _{\substack{u_{j M+2 i}^{j M+2 i-1} \\ i \in \Omega_{11}^{(j)}}}\left(T_{1}\right) \times \max _{\substack{u_{j M+2 i} \\ i \in \Omega_{11}^{(j)}}}\left(T_{2}\right) ;
$$

otherwise,

$$
\max _{u_{j M+1}^{j M+M}}(T)=\max _{\substack{u_{j M+2 i} \\ i \in \Omega_{01}^{(j)}}}\left(\max _{\substack{j M+2 i \\ u_{j M+2 i-1}^{j M} \\ i \in \Omega_{11}^{(j)}}}\left(T_{1}\right) \times \max _{\substack{u_{j M+2 i} \\ i \in \Omega_{11}^{(j)}}}\left(T_{2}\right)\right) .
$$

Under Assumption 1, considering Eq. [8], if $\Omega_{01}^{(j)}=\varnothing$, the maximal value of $T$ is just a product of the maximal value 
$\operatorname{Pr}\left(y_{1}^{4} \mid u_{1}^{4}\right)=\operatorname{Pr}\left(y_{1}^{2} \mid v_{1}, v_{2}\right) \times \operatorname{Pr}\left(y_{3}^{4} \mid u_{2}, u_{4}\right)$ where $v_{1}=u_{1} \oplus u_{2}$ and $v_{2}=u_{3} \oplus u_{4}$

(a)

$$
\text { DDDD : } \quad \max \left(\begin{array}{l}
\operatorname{Pr}\left(y_{1}^{4} \mid 0000\right), \\
\operatorname{Pr}\left(y_{1}^{4} \mid 0001\right), \\
\vdots \\
\operatorname{Pr}\left(y_{1}^{4} \mid 1110\right), \\
\operatorname{Pr}\left(y_{1}^{4} \mid 1111\right)
\end{array}\right)=\max \left(\begin{array}{l}
\operatorname{Pr}\left(y_{1}^{2} \mid 00\right), \\
\operatorname{Pr}\left(y_{1}^{2} \mid 01\right), \\
\operatorname{Pr}\left(y_{1}^{2} \mid 10\right), \\
\operatorname{Pr}\left(y_{1}^{2} \mid 11\right)
\end{array}\right) \times \max \left(\begin{array}{c}
\operatorname{Pr}\left(y_{3}^{4} \mid 00\right), \\
\operatorname{Pr}\left(y_{3}^{4} \mid 01\right), \\
\operatorname{Pr}\left(y_{3}^{4} \mid 10\right), \\
\operatorname{Pr}\left(y_{3}^{4} \mid 11\right)
\end{array}\right)
$$

(b)

For SC

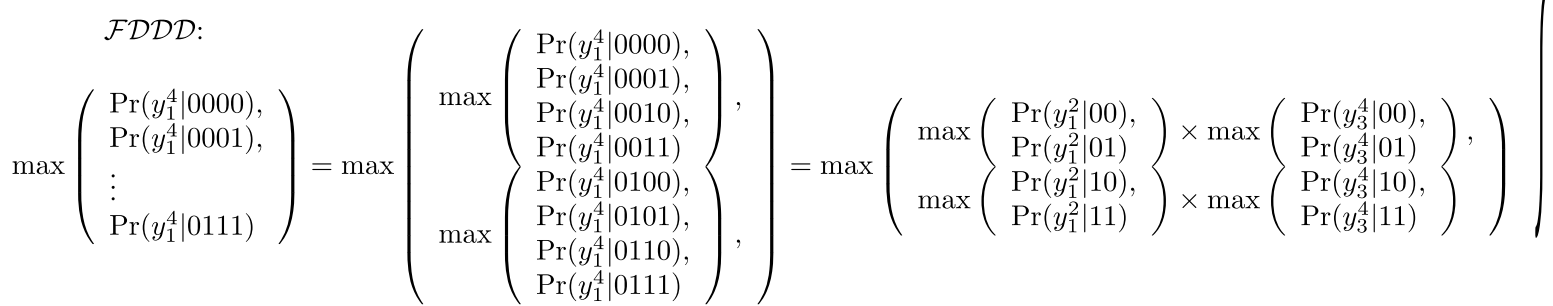

$$
\mathcal{D D D D}: \max _{2}\left(\begin{array}{l}
\operatorname{Pr}\left(y_{1}^{4} \mid 0000\right), \\
\operatorname{Pr}\left(y_{1}^{4} \mid 0001\right), \\
\vdots \\
\operatorname{Pr}\left(y_{1}^{4} \mid 1110\right), \\
\operatorname{Pr}\left(y_{1}^{4} \mid 1111\right)
\end{array}\right)=\max _{2}\left(\max _{2}\left(\begin{array}{l}
\operatorname{Pr}\left(y_{1}^{2} \mid 00\right), \\
\operatorname{Pr}\left(y_{1}^{2} \mid 01\right), \\
\operatorname{Pr}\left(y_{1}^{2} \mid 10\right), \\
\operatorname{Pr}\left(y_{1}^{2} \mid 11\right)
\end{array}\right) \otimes \max _{2}\left(\begin{array}{l}
\operatorname{Pr}\left(y_{3}^{4} \mid 00\right), \\
\operatorname{Pr}\left(y_{3}^{4} \mid 01\right), \\
\operatorname{Pr}\left(y_{3}^{4} \mid 10\right), \\
\operatorname{Pr}\left(y_{3}^{4} \mid 11\right)
\end{array}\right)\right)
$$

(d)

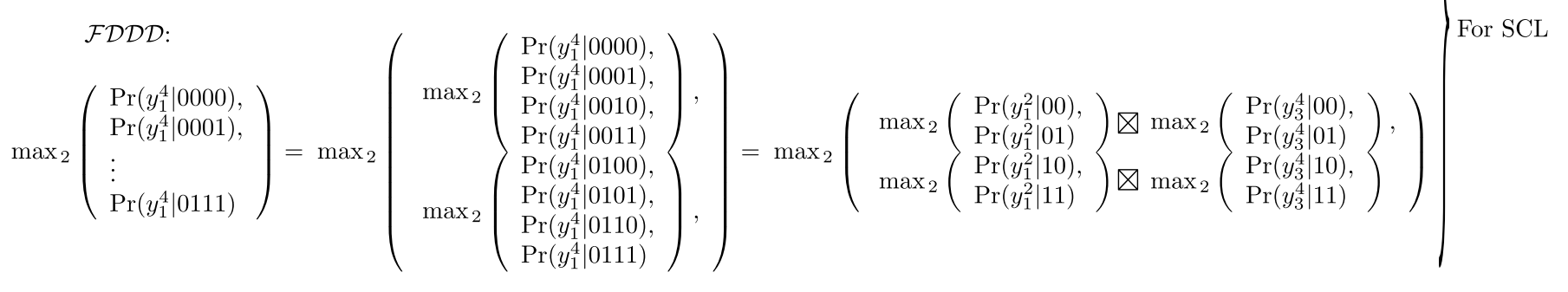

(e)

Fig. 1. Examples for calculating $\max ()$ and $\max _{2}()$ functions when $M=4, N=4, q=2$, and $j=0$, (a) the calculation rule for $\operatorname{Pr}\left(y_{1}^{4} \mid u_{1}^{4}\right)$, (b) the calculation of $\max \left(\operatorname{Pr}\left(y_{1}^{4} \mid u_{1}^{4}\right)\right)$ in [23] when the frozen-location pattern is ' $\mathcal{D} \mathcal{D} \mathcal{D} \mathcal{D}$ ', (c) the calculation of $\max \left(\operatorname{Pr}\left(y_{1}^{4} \mid u_{1}^{4}\right)\right)$ in $23 \mid$ when the frozen-location pattern is ' $\mathcal{F D D D D}$ ', (d) the calculation of $\max _{2}\left(\operatorname{Pr}\left(\mathrm{y}_{1}^{4} \mid \mathrm{u}_{1}^{4}\right)\right)$ when the frozen-location pattern is ' $\mathcal{D} \mathcal{D} \mathcal{D} \mathcal{D}^{\prime}$, and (e) the calculation of $\max _{2}\left(\operatorname{Pr}\left(\mathrm{y}_{1}^{4} \mid \mathrm{u}_{1}^{4}\right)\right)$ when the frozen-location pattern is ' $\mathcal{F D D D}$ '.

of $T_{1}$ and the maximal value of $T_{2}$. For example, Fig. 1 b) shows an example of frozen-location pattern ' $\mathcal{D D D} \mathcal{D}$ ' which has $\Omega_{01}^{(0)}=\varnothing$, when $M=4$ and $N=4$. If $\Omega_{01}^{(j)}$ is not empty, for any $i \in \Omega_{01}^{(j)}, v_{\frac{j M}{2}+i}=u_{j M+2 i}$.

\section{B. Divide-and-conquer AML decoding unit for SCL-based algorithms}

Extending the idea in Eqs. (8) and (9), we propose a divideand-conquer AML decoding method for SCL-based algorithms under Assumption 1. For SC-based algorithms, only the maximal value of $\operatorname{Pr}\left(\mathbf{y}, \hat{u}_{1}^{j M} \mid u_{j M+1}^{j M+M}\right)$ is needed. In contrast, for SCL-based algorithms with list size $L$, the $L$ maximal values of $\operatorname{Pr}\left(\mathbf{y}, \hat{u}_{1}^{j M} \mid u_{j M+1}^{j M+M}\right)$ are needed. A simple understanding for our method is that the $\max (\operatorname{Pr}(\rho))$ function is replaced by a function finding the $L$ maximal values of $\operatorname{Pr}(\rho)$, denoted as $\left[\operatorname{Pr}\left(\rho_{1}\right), \cdots, \operatorname{Pr}\left(\rho_{L}\right)\right]=\max _{L}(\operatorname{Pr}(\rho)) \cdot \max _{L}(\operatorname{Pr}(\rho)) \otimes$ $\max _{L}(\operatorname{Pr}(\psi))$ generates $L^{2}$ values of $\operatorname{Pr}\left(\rho_{i}\right) \operatorname{Pr}\left(\psi_{j}\right)$ for $1 \leq$ $i, j \leq L$.
The path expansion-and-pruning procedure of SCL-based algorithms is divided into two stages. In the first stage, the $q$ most reliable paths are selected for each list by calculating and comparing path metrics. In the second stage, the $L$ most reliable paths among the $q L$ survival paths generated in the first stage. This two stage approach was proposed in our prior work [19], and the novelty herein is that we use the divideand-conquer method to reduce the complexity of the first stage. The second stage has been described in [19], and we omit its discussions.

Assuming $\left|\Omega_{01}^{(j)}\right|=\beta_{j},\left|\Omega_{11}^{(j)}\right|=\gamma_{j}$. When $\beta_{j} \geq 1$, let $\Omega_{01}^{(j)}=\left\{i_{1}^{(j)}, i_{2}^{(j)}, \cdots, i_{\beta_{j}}^{(j)}\right\}\left(i_{1}^{(j)}<i_{2}^{(j)}<\cdots<i_{\beta_{j}}^{(j)}\right)$. The first stage includes:

Step 0: the RCC method [19] is applied to calculate both $T_{1}$ and $T_{2}$.

Step 1: Given any $\beta_{j}$-bit binary vector $\mathcal{B}^{(j)}=$ $\left(u_{j M+2 i_{1}^{(j)}}, u_{j M+2 i_{2}^{(j)}}, \ldots, u_{j M+2 i_{\beta_{j}}^{(j)}}\right)$, there are $2^{\gamma_{j}}$ possible values for both $v_{\frac{j M}{2}+1}^{\frac{j M+M}{2}}$ and $u_{j M+1, e}^{j M+M}$. We find the $\min \left(q, 2^{\gamma_{j}}\right)$ 
maximal values of $2^{\gamma_{j}}$ values of $T_{1}$, and the $\min \left(q, 2^{\gamma_{j}}\right)$ maximal values of $2^{\gamma_{j}}$ values of $T_{2}$.

Step 2: For $\mathcal{B}^{(j)}$, there are $\left(\min \left(q, 2^{\gamma_{j}}\right)\right)^{2}$ values of $T$, which is a product of values of $T_{1}$ and $T_{2}$ generated in Step 1.

Step 3: The $q$ maximal values are selected from $\left(\min \left(q, 2^{\gamma_{j}}\right)\right)^{2} 2^{\beta_{j}}$ values of $T$ generated by Step 2 because there are $2^{\beta_{j}}$ possible values for $\mathcal{B}^{(j)}$.

If $\Omega_{01}^{(j)}=\varnothing$ and $\beta_{j}=0$, we still use the aforementioned four steps to find the $q$ most reliable paths for each list except that $\mathcal{B}^{(j)}$ is considered as a void binary vector which is the only value for $\mathcal{B}^{(j)}$ when $\beta_{j}=0$.

Fig. 1(d) and 1(e) show two examples for frozen-location patterns ' $\mathcal{D} \mathcal{D D D}$ ' and ' $\mathcal{F D D D}$ ', respectively when $M=4$, $N=4$, and $q=2$. After these four steps are carried out for each list, there are $q L$ values of $T$ left, which are sorted to choose the $L$ maximal values in the second stage.

The proposed divide-and-conquer AML decoding unit has a lower computational complexity. It reduces the number of symbol-wise channel transition probabilities dealt by the list pruning function by sorting the intermediate calculation results generated by the RCC method [19], whereas the DMM, RCC, and DRH methods perform list pruning function after all the symbol-wise channel transition probabilities are calculated. For example, in Fig. 1(d), the DMM, RCC, and DRH methods perform $\max _{2}\left(\operatorname{Pr}\left(y_{1}^{4} \mid u_{1}^{4}\right)\right)$ after all 16 values of $\operatorname{Pr}\left(y_{1}^{4} \mid u_{1}^{4}\right)$ are calculated. The proposed divide-and-conquer method performs $\max _{2}\left(\operatorname{Pr}\left(y_{1}^{2} \mid v_{1}^{2}\right)\right)$ and $\max _{2}\left(\operatorname{Pr}\left(y_{3}^{4} \mid u_{2}, u_{4}\right)\right)$ first. Then it finds the two maximal values out of four elements generated by $\max _{2}\left(\operatorname{Pr}\left(y_{1}^{2} \mid v_{1}^{2}\right)\right) \otimes \max _{2}\left(\operatorname{Pr}\left(y_{3}^{4} \mid u_{2}, u_{4}\right)\right)$. The output of the proposed AML decoding unit is the same as those of other ML decoding units if they have the same input.

Given an $M$-bit symbol $u_{j M+1}^{j M+M}, \quad \Omega_{01}^{(j)}, \quad \Omega_{11}^{(j)}$, $\operatorname{Pr}\left(y_{1}^{\frac{N}{2}}, \hat{v}_{1}^{\frac{j M}{2}} \mid v_{\frac{j M}{2}+1}^{\frac{j M+M}{2}}\right)$, and $\operatorname{Pr}\left(y_{\frac{N}{2}+1}^{N}, \hat{u}_{1, e}^{j M} \mid u_{j M+1, e}^{j M+M}\right)$, the first stage using the divide-and-conquer decoding unit needs two $2^{\gamma_{j}}$-to- $\left(\min \left(q, 2^{\gamma_{j}}\right)\right)$ sorts, one $\left(\min \left(q, 2^{\gamma_{j}}\right)\right)^{2} 2^{\beta_{j}}$-to- $q$ sort, and $\left(\min \left(q, 2^{\gamma_{j}}\right)\right)^{2} 2^{\beta_{j}}$ multiplications per list, whereas the ML decoding unit in [19] needs $2^{\beta_{j}+2 \gamma_{j}}$ multiplications and a $2^{\beta_{j}+2 \gamma_{j}}$-to- $q$ sort per list. By examining all possible values of $\beta_{j}$ and $\gamma_{j}$, we can find the worst-case computational complexity.

We demonstrate the advantage of the proposed divide-andconquer AML unit in computational complexity as opposed to other ML decoding units with an example of $M=8$ and $q=$ 4. Henceforth, we only discuss the computational complexity per list to accomplish the job of the first stage of the proposed method. Table $\Pi$ lists worse-case computational complexities of different methods and shows that the proposed method has the smallest computational complexity when 81 eight-bit frozenlocation patterns under Assumption 1 are needed to be dealt with.

Regarding the impact on the error performance, our proposed method has the same performance degradation as in [25]. If $q \geq L$, our method does not introduce any performance degradation for SCL-based algorithms. If $q<L$, the performance degradation depends on values of $q$ and $L$, and the performance degradation is usually negligible when $q$ and $L$
TABLE I

WORST-CASE COMPUTATIONAL COMPLEXITY OF DIFFERENT METHODS WHEN $M=8$ AND $q=4$.

\begin{tabular}{|c|l|}
\hline Method & Computational Complexity \\
\hline RCC $\left[\begin{array}{ll}25 \\
\text { DMM }\end{array}\right.$ & 304 multiplications, a 256-to-4 sort \\
\hline DRH & 1792 multiplications, a 256-to-4 sort \\
\hline $\begin{array}{c}\text { Divide-and-Conquer } \\
\text { AML }\end{array}$ & $\begin{array}{l}\text { 112 multiplications, a 64-to-4 sort } \\
\text { and two 16-to-4 sorts }\end{array}$ \\
\hline $\begin{array}{c}\text { Divide-and-Conquer } \\
\text { AML }{ }^{\dagger}\end{array}$ & $\begin{array}{l}\text { 80 multiplications, a 32-to-4 sort } \\
\text { and two 16-to-4 sorts }\end{array}$ \\
\hline \hline LC-AML * & $\begin{array}{l}\text { 80 multiplications, a 32-to-4 sort } \\
\text { and four 8-to-4 sorts }\end{array}$ \\
\hline
\end{tabular}

$\ddagger$ All 81 eight-bit patterns under Assumption 1 are dealt with.

$\dagger$ Only nine eight-bit patterns in Sec. IV-A are dealt with.

* Only six eight-bit patterns in Sec. V are dealt with.

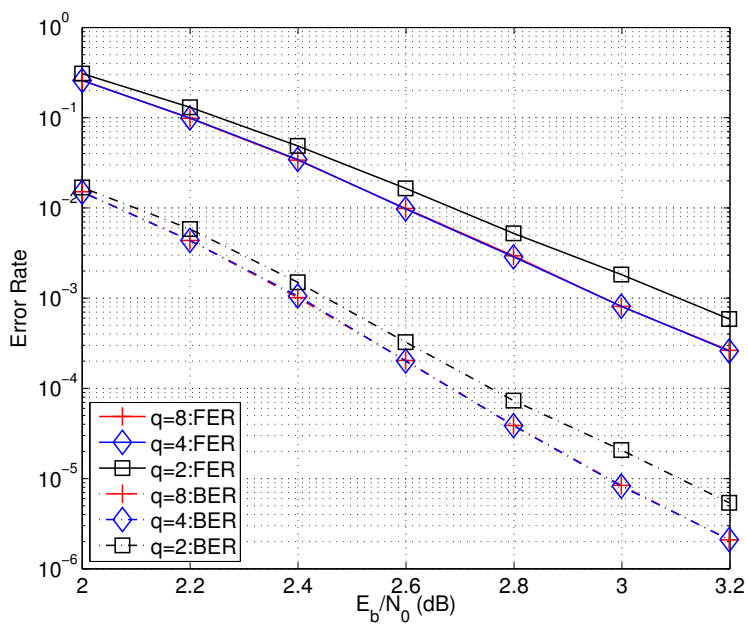

Fig. 2. Frame and bit error rates of CA-SCL decoders with different $q$ s for a $(2048,1433)$ polar codes with a 32-bit CRC over the AWGN channel when adapting the LC-AML decoding unit.

are small. Fig. 2 shows the frame and bit error rates of a (2048, 1433) polar code with a 32-bit CRC of CA-SCL decoders with the LC-AML decoding unit when $M=8$ and $L=8$. When $q=4$, the performance loss is negligible. However, $q=2$ leads to a performance loss of about $0.1 \mathrm{~dB}$ at an FER level of $10^{-3}$.

\section{Frozen-Location Patterns for POLAR CODES}

Considering the hardware implementation for the divideand-conquer AML unit, a uniform hardware design for all frozen-location patterns is preferred rather than different dedicated designs for various frozen-location patterns. For $M=8$ and $M=16$, there are 81 and 6561 possible frozen-location patterns satisfying Assumption 1, respectively. Actually, some of them may never exist in a polar code. Therefore, we want to know the exact number of frozen-location patterns in a polar code, since the number of frozen-location patterns impacts the complexity of the divide-and-conquer AML decoding unit for SCL-based algorithms: the more frozen-location patterns, the higher complexity the divide-and-conquer AML decoding unit has. 


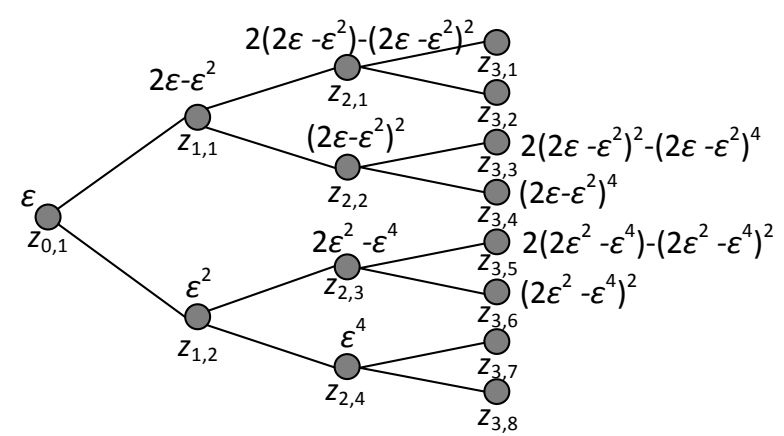

Fig. 3. Recursive calculation of erasure probabilities for polar codes over the BEC when the code length is no more than eight.

\section{A. Polar Codes for the BEC}

For polar codes constructed for the BEC with an erasure probability $\epsilon(0<\epsilon<1)$, Eqs. (1) and (2) are used in [28]. Fig. 3 illustrates the transition graph of the erasure probability for constructing a polar code with the code length no more than eight, and also can be viewed as a sub-graph of the erasure probability transition for any eight-bit symbol of a polar code because of the recursive calculation. In order to examine frozen-location patterns in a polar code, we have following results regarding the ordering of $z_{i, j}$ for $i \geq 1$ and $1 \leq j \leq 2^{i}$. This ordering determines possible frozen-location patterns in a polar code.

Proposition 1. Assuming $z_{0,1}=\epsilon \in(0,1)$, given any $i \geq 1$ and $1 \leq j \leq 2^{i}, z_{i, j}$ is calculated by Eqs. (1) or (2). We have

(a) $0<z_{i, j}<1$ for $i \geq 1$ and $1 \leq j \leq 2^{i}$,

(b) $z_{i, 2 j-1}>z_{i, 2 j}$ for $i \geq 1$ and $1 \leq j \leq 2^{i-1}$,

(c) $z_{i, 4 j-3}>z_{i, 4 j-2}>z_{i, 4 j-1}>z_{i, 4 j}$ for $i \geq 2$ and $1 \leq$ $j \leq 2^{i-2}$

(d) $z_{i, 8 j-7}>z_{i, 8 j-6}>z_{i, 8 j-5}>z_{i, 8 j-3}>z_{i, 8 j-4}>$ $z_{i, 8 j-2}>z_{i, 8 j-1}>z_{i, 8 j}$ for $i \geq 3$ and $1 \leq j \leq 2^{i-3}$.

Proof of Prop. 1 is provided in the Appendix.

Now, let us explain how the ordering of $z_{n, j}$ determines $2^{m}$-bit $(1 \leq m \leq 3)$ frozen-location patterns in an $(N, K)$ polar code over the BEC. First, to choose elements of $\mathcal{A}^{c}$ for an $(N, K)$ polar code over the BEC, $\mathcal{A}^{c}$ is chosen such that $\sum_{j \in \mathcal{A}^{c}} z_{n, j}$ is maximal and $\left|\mathcal{A}^{c}\right|=N-K$, where $N=2^{n}$. Then, if there are $k_{j}$ frozen bits in a symbol $u_{2^{m} j+1}^{2^{m}(j+1)}(0 \leq$ $\left.j<\frac{N}{2^{m}}\right)$, a set $\mathcal{A}_{j}^{c}$ consisting of indexes of these $k_{j}$ frozen bits must be chosen such that $\sum_{t \in \mathcal{A}_{j}^{c}} z_{n, t}$ is maximal while $\left|A_{j}^{c}\right|=k_{j}$. For example, assuming there are four frozen bits in $u_{1}^{8}$ in a $(16,12)$ polar code, by Proposition $1(\mathrm{~d}), z_{4,1}>z_{4,2}>$ $z_{4,3}>z_{4,5}>z_{4,4}>z_{4,6}>z_{4,7}>z_{4,8}$. Hence, $u_{1}, u_{2}, u_{3}$, and $u_{5}$ will be frozen bits and the frozen-location pattern for $u_{1}^{8}$ will be ' $\mathcal{F} \mathcal{F} \mathcal{F} \mathcal{D} \mathcal{F} D \mathcal{D D}$ '.

Therefore, for polar codes constructed by the method in [28], by Proposition 11(b), there are three two-bit frozenlocation patterns: ' $\mathcal{D D}$ ', ' $\mathcal{F D}$ ', and ' $\mathcal{F F}$ '. We note that the implication of Proposition 1 (b) is the counterpart over the BEC of Assumption 1 in [23]. By Proposition 11(c), there are five four-bit frozen-location patterns: ' $\mathcal{D D D D}$ ', ' $\mathcal{F D D D}$ ', ' $\mathcal{F} \mathcal{F} \mathcal{D D}$ ', ' $\mathcal{F} \mathcal{F} \mathcal{F} \mathcal{D}^{\prime}, \quad$ and ' $\mathcal{F} \mathcal{F} \mathcal{F} \mathcal{F}$ '. By Proposition 1]d), there are nine eight-bit frozen-location patterns:

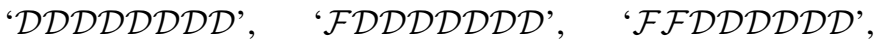
' $\mathcal{F F} \mathcal{F} \mathcal{D D D D D}$ ', ' $\mathcal{F} \mathcal{F} \mathcal{F} \mathcal{D} \mathcal{F} D \mathcal{D D}$ ', ' $\mathcal{F} \mathcal{F} \mathcal{F} \mathcal{F} \mathcal{F D D D}$ ', ' $\mathcal{F F} \mathcal{F} \mathcal{F} \mathcal{F} \mathcal{F D D}$ ', ' $\mathcal{F} \mathcal{F} \mathcal{F} \mathcal{F} \mathcal{F} \mathcal{F} \mathcal{F}$ ', and ' $\mathcal{F} \mathcal{F} \mathcal{F} \mathcal{F} \mathcal{F} \mathcal{F} \mathcal{F} \mathcal{F}$ '.

For a larger symbol size, it is hard to get the ordering of $z_{i, j}$ by an analytical method. A numerical method can be used. For example, the symbol size is 16. By Proposition 11(d), we have $z_{4,1}>z_{4,2}>z_{4,3}>z_{4,5}>z_{4,4}>z_{4,6}>z_{4,7}>z_{4,8}$ and $z_{4,9}>z_{4,10}>z_{4,11}>z_{4,13}>z_{4,12}>z_{4,14}>z_{4,15}>z_{4,16}$. We also have $z_{4,5}>z_{4,9}>z_{4,7}>z_{4,11}$ and $z_{4,4}>z_{4,6}>$ $z_{4,10}>z_{4,8}>z_{4,12}$. For $0<z_{0,1}=\epsilon<1$,

$\left.z_{4,10}-z_{4,7}=2 \epsilon^{4}(\epsilon-1)^{4}\left[\epsilon^{3}\left(\epsilon^{2}-\epsilon+24\right)(\epsilon-1)^{3}-8\right)\right]<0$.

Moreover, for $0<z_{0,1}=\epsilon<1, z_{4,4}-z_{4,9}=2 \epsilon^{2}(\epsilon-$ $1)^{4}\left(\epsilon^{10}-4 \epsilon^{9}+34 \epsilon^{8}-116 \epsilon^{7}+237 \epsilon^{6}-375 \epsilon^{5}+420 \epsilon^{4}-280 \epsilon^{3}+\right.$ $\left.102 \epsilon^{2}-16 \epsilon-4\right)<0$ and $z_{4,8}-z_{4,13}=2 \epsilon^{4}(\epsilon-1)^{2}\left(\epsilon^{10}-6 \epsilon^{9}+\right.$ $\left.43 \epsilon^{8}-132 \epsilon^{7}+251 \epsilon^{6}-262 \epsilon^{5}+121 \epsilon^{4}-8 \epsilon^{3}-6 \epsilon^{2}-4 \epsilon-2\right)<0$. These two inequalities are verified numerically.

Because of the recursive calculation of $z_{i, j}$, for $i \geq 4$ and $1 \leq j \leq 2^{i-4}$, we have

$$
\begin{aligned}
z_{i, 16 j-15} & >z_{i, 16 j-14}>z_{i, 16 j-13}>z_{i, 16 j-11} \\
& >z_{i, 16 j-7}>z_{i, 16 j-12}>z_{i, 16 j-10}>z_{i, 16 j-9} \\
& >z_{i, 16 j-6}>z_{i, 16 j-5}>z_{i, 16 j-3}>z_{i, 16 j-8} \\
& >z_{i, 16 j-4}>z_{i, 16 j-2}>z_{i, 16 j-1}>z_{i, 16 j} .
\end{aligned}
$$

Thus, there are only 17 frozen-location patterns for 16-bit symbols.

It is not meaningful to consider the symbol size greater than 16 , because this will incur very high complexity for hardware implementations.

\section{B. Polar Codes for the AWGN Channel}

For the construction method introduced in [29] for the AWGN channel, it is difficult to analyze the relationship between $z_{3, i}$ 's for $1 \leq i \leq 8$ based on Eqs. (3) and (5). Instead, we examine eight polar codes constructed with the method in [29], which have code lengths from $2^{10}$ to $2^{13}$ and code rates of 0.5 and 0.8 to identify eight-bit frozen-location patterns. By examining all eight-bit symbols of these polar codes, we found that in these codes there are only nine eightbit frozen-location patterns, which are the same as those for polar codes constructed for the BEC, listed in Sec. IV-A Our observation is consistent with Assumption 1 in [23].

\section{Computational Complexity of the Divide-and-Conquer AML Decoding Unit}

When it needs to deal with only the frozen-location patterns mentioned in Sections IV-A and IV-B, the divide-and-conquer AML decoding unit has a smaller complexity. If $M=8$ and $q=4$, it needs 80 multiplications, two 16-to-4 sorts, and a 32-to-4 sort, as listed in Table I. It saves 32 multiplications, a 32-to-4 sort and a 8-to-4 sort compared with the divide-andconquer AML decoding unit which deals with all 81 frozenlocation patterns following Assumption 1, since a 64-to-4 sort consists of two 32-to-4 sorts and a 8-to-4 sort. 
If $M=16$ and $q=4$, to deal with all $3^{8}=6561$ 16-bit frozen-location patterns satisfying Assumption 1, the first stage of the proposed ML decoding unit needs 1632 multiplications, two 256-to-4 sorts, and a 1024-to-4 sort. However, to deal with 17 16-bit frozen-location patterns discussed in Section IV-A. the simplified divide-and-conquer AML decoding unit needs 736 multiplications, two 256 -to-4 sorts, and a 128-to-4 sort.

\section{Low-Complexity AML Decoding Unit}

For convenience, we implement the proposed divide-andconquer AML decoding unit, assuming $M=8$ henceforth. Our implementation can be readily extended to other values of $M$. To further reduce complexity and latency, we do not use the divide-and-conquer method to deal with patterns ' $\mathcal{D D D D D D D D}$ ', ' $\mathcal{F} \mathcal{F} \mathcal{F} \mathcal{F} \mathcal{F} \mathcal{F} \mathcal{F} D$ ', and ' $\mathcal{F} \mathcal{F} \mathcal{F} \mathcal{F} \mathcal{F} \mathcal{F} \mathcal{F} F$ ', which will be described in Sec. V-B Then the divide-andconquer AML decoding unit can be simplified further by dealing with only the remaining six eight-bit frozen-location patterns. This simplified divide-and-conquer AML decoding unit, referred to as the LC-AML decoding unit, needs 80 multiplications, four 8-to-4 sorts, and a 32-to-4 sort. It saves two 8-to-4 sorts compared with the divide-and-conquer AML decoding unit dealing with nine patterns, since a 16-to-4 sort consists of three 8-to- 4 sorts. This also leads to a shorter critical path in our design than the divide-and-conquer AML decoding unit.

\section{A. Hardware Design for the LC-AML Decoding Unit}

SCL-based polar decoders in the literature can be divided into two categories: the log-likelihood (LL) based decoders [11], [25], [30] and the log-likelihood-ratio (LLR) based decoders [10], [27]. Although our proposed algorithm in Sec. III] is described in the probability domain, it can be easily adapted for both the LL-based decoder and the LLR-based decoder. We focus on the LLR-based polar decoder, because in general the LLR-based decoder has a better area efficiency than the LL-based decoder.

First, we adapt the proposed LC-AML decoding unit to the LLR-based SCL decoder. Given path metrics $\mathrm{PM}_{k}^{(t)}$ of $L$ list survivors and assuming $u_{t}$ is the last bit processed by the decoder, where $1 \leq k \leq L, 1 \leq t \leq N$, and $t$ is a multiple of $M$. Suppose $\alpha_{j, l}(0 \leq j<M)$ represents the LLR of $\operatorname{Pr}\left(y_{j \frac{N}{M}+1}^{(j+1) \frac{N}{M}}, \hat{w}_{1+j \frac{t}{M}+j \frac{N}{M}}^{\frac{N}{M}} \mid w_{\frac{t}{M}+1+j \frac{N}{M}}\right)$ corresponding to the list $l$. The path metric $\mathcal{P} \mathcal{M}_{k, p}^{(t+M)}$ of the $p$-th expanded path from the $k$-th list survivor corresponding to $u_{t+1}^{t+M}=p\left(0 \leq p<2^{M}\right)$ is $\mathcal{P M}_{k, p}^{(t+M)}=\mathrm{PM}_{k}^{(t)}+\sum_{j=0}^{M-1} m_{j}\left|\alpha_{j, l}\right|$, where $m_{j}=0$ if $w_{\frac{t}{M}+1+j \frac{N}{M}}=\frac{1}{2}\left(1-\operatorname{sign}\left(\alpha_{j, l}\right)\right)$ [10]. Otherwise, $m_{j}=1$. Then our goal is to calculate $\mathcal{P} \mathcal{M}_{k, p}^{(t+M)}$ and to select the $L$ minimum values of $\mathcal{P} \mathcal{M}_{k, p}^{(t+M)}$.

Fig. 4 shows the top architecture of our low-complexity implementation for the LC-AML decoding unit. MLD_S1 calculates path metrics and selects the $q$ minimum values for each list. FrzInfVec is an $M$-bit frozen bit indication vector $\left(f_{1}, f_{2}, \cdots, f_{M}\right)$ for $u_{t+1}^{t+M}$. For $1 \leq j \leq M$, if $u_{t+j}$ is a frozen bit, $f_{j}=1$; otherwise, $f_{j}=0$. LLRInV $\_l$ is the vector $\left(\alpha_{0, l}, \alpha_{1, l}, \cdots, \alpha_{M-1, l}\right)$ for $1 \leq l \leq L$.

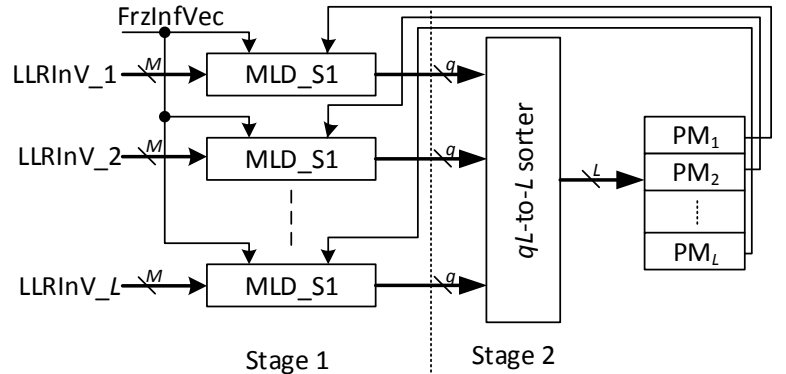

Fig. 4. Top architecture of the proposed LC-AML decoding unit.

Fig. 5(a) shows the design for MLD_S1_q4 when $M=8$ and $q=4$. Here, we focus on the data path for calculating path metrics. The circuitry to generate symbol values associated with path metrics is simple and consists of XORs, and therefore is omitted. The data paths corresponding to different steps aforementioned in Section III are labeled as well.

In Step 0, two RCC blocks, shown in Fig. 5(b), are used. LLR $a_{i}(16 \leq i \leq 31)$ associated with $\operatorname{Pr}\left(y_{\frac{N}{2}+1}^{N}, \hat{u}_{1, e}^{8 j} \mid u_{8 j+1, e}^{8 j+8}=(i-16)_{2}\right)$ is calculated by the right RCC block. LLR $a_{i}(0 \leq i \leq 15)$ associated with $\operatorname{Pr}\left(y_{1}^{\frac{N}{2}}, \hat{v}_{1}^{4 j} \mid v_{4 j+1}^{4 j+4}=(i)_{2}\right)$ is calculated by the left RCC block. Here, $(i)_{2}$ represents the binary string of interger $i$. 16ADDER contains 16 adders to calculate path metrics, shown in Fig. 5. c).

In Step 1, for different frozen-location patterns, path metrics go through different data paths selected by 16 2-to- 1 multiplexers. Their control words are 1 if frozen-location patterns are $\mathcal{F} \mathcal{D D D D D D D}, \mathcal{F} \mathcal{F D D D D D D}$; otherwise they are 0 .

In Step 2, results from Step 1 are combined to calculate $\sum_{j=0}^{7} m_{j}\left|\alpha_{j, l}\right|$.

In Step 3, there are 32 path metrics going through a 32-to-4 sorter. However, for some frozen-location patterns, the number of valid symbol values is less than 32 because the number of frozen bits can be larger than three. Therefore, path metrics associated with those invalid symbol values need to be set to the maximal positive value as well so that the 4 minimum path metrics belong to valid symbol values. MSNG accomplishes this job with FrzInfVec, which contains the frozen-location pattern information.

Different sorters used in our design are shown in Fig. 5 (d), 5(e) and 5(f). S8TO4 finds the minimum four values of eight values. S4 sorts the four inputs and outputs them in decreasing order and has a shorter critical path of two comparators and one 4-to-1 multiplexer than a four-input bitonic sorter [31], which has a critical path of three comparators. S32To4 consists of seven S8TO4 units in a binary tree structure.

Although MLD_S1_q4 is designed for six eight-bit frozenlocation patterns, other frozen-location patterns also can be dealt with by MLD_S1_q4, such as all frozen-location patterns satisfying the following two conditions. First, the frozenlocation pattern has at least three ' $\mathcal{F}$ 's. Second, two frozen bits are located at the first two bits of the data symbol. 


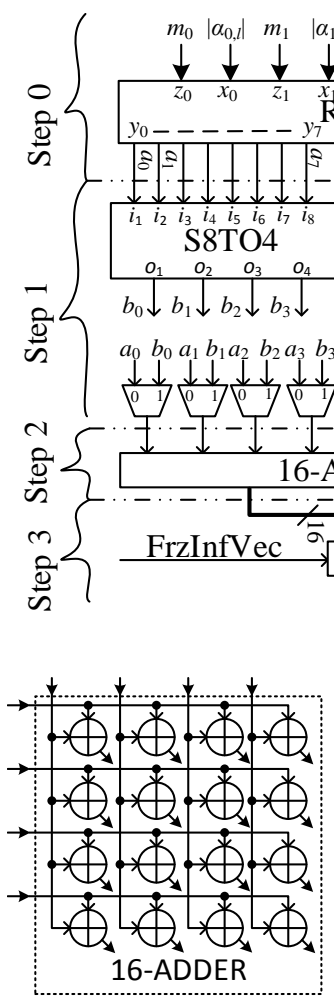

(c)

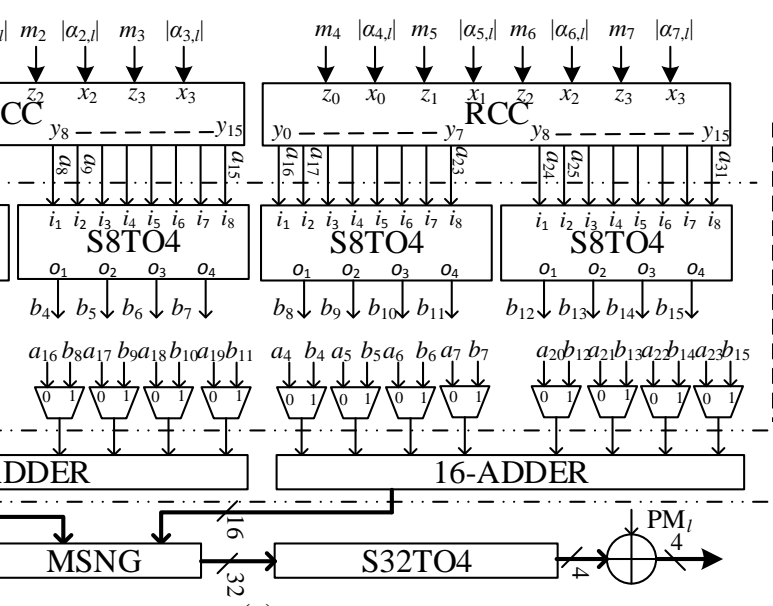

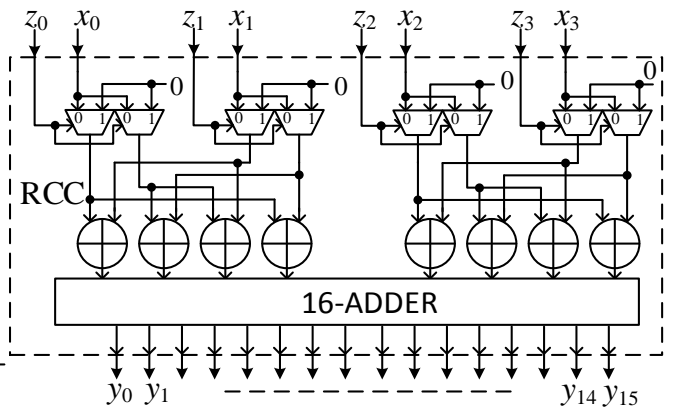

(b) (a)

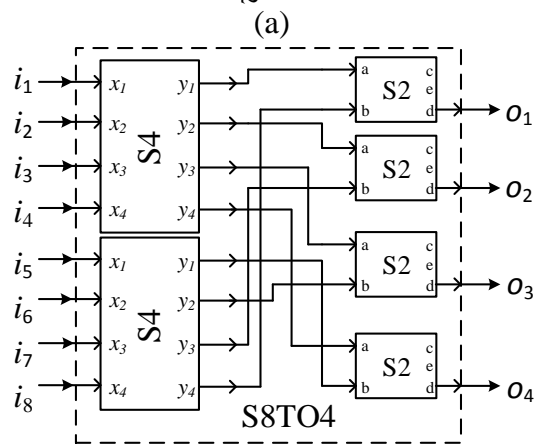

(d)

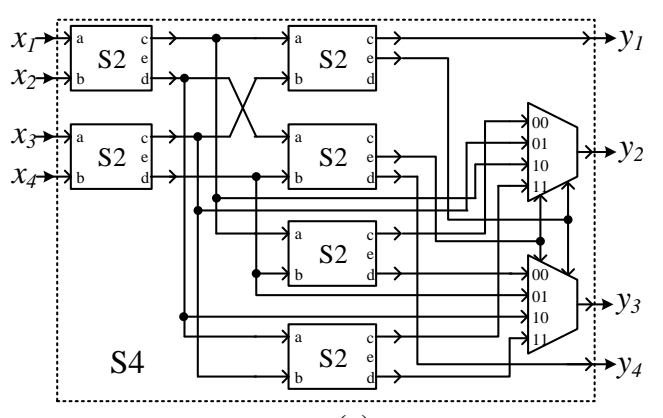

(e)

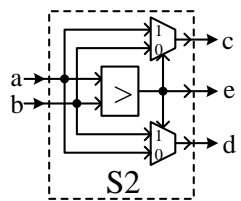

(f)

Fig. 5. Design of MLD_S1_q4 when $M=8$ and $q=4$. (a) top architecture of MLD_S1_q4, (b) diagram of RCC, (c) diagram of 16-ADDER, (d) diagram of $\mathrm{S} 8 \mathrm{TO} 4$, (e) diagram of $\mathrm{S} 4$, (f) diagram of $\mathrm{S} 2$.

\section{B. Area-Efficient SCL Decoder}

To examine the advantage of our proposed design, we incorporate MLD_S1_q4 into CA-SCL polar decoders with list size $L=4$. Architecture-wise, our decoder, referred to as the AE-SCL decoder, is almost the same as the architecture of the tree based reduced latency SCL polar decoder in [27], which performs the CA-SCL decoding algorithm on a binary tree representation of a polar code, except that our AE-SCL decoder uses the LC-AML decoding unit instead of the DRH ML decoding unit used in [27].

Leaf nodes of the decoding tree for our decoder are divided into four categories:

1) Rate-0 node: its frozen-location pattern contains only ' $\mathcal{F}$ ', i.e., the node contains only frozen bits.

2) Rate-1 node: its frozen-location pattern contains only ' $\mathcal{D}$ ', i.e., the node contains only information bits.

3) Repetition node [13]: its frozen-location pattern is either ' $\mathcal{F} \mathcal{F} \mathcal{F} \mathcal{F} \mathcal{F} \mathcal{F} \mathcal{F} \mathcal{F}_{\mathcal{F}} \mathcal{F} \mathcal{F} \mathcal{F} \mathcal{F} \mathcal{F} \mathcal{F}$ ' or ' $\mathcal{F} \mathcal{F} \mathcal{F} \mathcal{F} \mathcal{F} \mathcal{F} \mathcal{F}$ '.

4) Rate-R-2 node: its frozen-location pattern is one of the six eight-bit frozen-location patterns.

Rate- 0 and rate- 1 nodes are decoded with the same methods as in [27]. The main difference between our proposed decoder here and the tree based reduced latency SCL polar decoder is how to deal with repetition nodes and rate-R-2 nods. For repetition nodes, a binary tree of adders is used to calculate LLRs in order to reduce the decoding latency [13]. Rate-R-2 nodes are dealt with by MLD_S1_q4, which reduces the area of AE-SCL decoders.

\section{Synthesis Results}

AE-SCL decoders with $L=4$ are implemented for three polar codes: a $(1024,512)$ code, an $(8192,4096)$ code, and a $(32768,29504)$ code. These three codes are with a 32-bit cyclic redundancy check. The number of processing units of decoders for $N=1024$ is 256 . For the other two codes, the decoder has 512 processing units. Five-bit channel LLRs are used. The synthesis tool is Cadence RTL compiler. The process technology is TSMC 90nm CMOS technology. Here, four stages of pipeline registers are used in the LC-AML decoding unit. Areas of different ML decoding units for the $(1024,512)$ polar codes are listed in Table III The area of our proposed LC-AML decoding unit is only one fourth of that of the ML decoding unit in [27]. By taking into account fewer patterns, the area of the LC-AML decoding unit is $67 \%$ of that of the Divide-and-Conquer AML design which deals with all 81 eight-bit frozen-location patterns following Assumption 1.

The synthesis results of three entire decoders (AE-SCLs) are also listed in Tables V, VI, and VII respectively. Here, NIT means the net information throughput. Compared with decoders in [27], the SCL decoder architecture with the best area efficiency to our knowledge, the AE-SCL decoders have smaller areas because the proposed LC-AML decoding unit is applied. The LC-AML decoding unit has a slightly larger decoding latency than that in [27], because the proposed LC- 
TABLE II

AREAS OF DIFFERENT ML DECODING UNITS FOR THE $(1024,512)$ POLAR CODE.



AML decoding unit deals with only eight-bit frozen-location patterns, whereas the ML decoding unit in [27] can deal with some 16-bit frozen-location patterns. Since the extra decoding cycles needed by AE-SCL decoders are a very small fraction of the entire decoding cycles, the proposed AE-SCL decoders still achieve better area efficiency than decoders in [27]. For example, for the $(1024,512)$ polar code, the area efficiency of the AE-SCL decoder is 1.93 times of that of the decoder in [27]. As the code length increases, the advantage of area efficiency is less because the ML decoding unit occupies a smaller fraction of the entire decoder if the code is longer. Compared with symbol-decision SCL decoders in [10], [24], [25], the advantage of our decoders on the area efficiency is more significant. The area efficiency of the AE-SCL decoder is 3.32, 8.25, and 3.17 times of that of decoders in [10], [24], [25], respectively, for the $(1024,512)$ polar code.

\section{Multi-Mode SCL DeCoder}

All existing SCL polar decoders in the literature provide fixed throughput and decoding latency given a polar code. These SCL decoders cannot adapt to variable communication channels and applications. In order to adapt to different throughput and latency requirements, we propose a multimode SCL (MM-SCL) decoder with $n_{d}$ decoding paths, which can decode $P$ received words with list size $L$ in parallel, where $1 \leq P, L \leq n_{d}$ and $n_{d} \geq P \times L$. This multi-mode feature requires the decoder to perform SCL decoding algorithms with different list sizes (the SC decoding algorithm is a special case of the SCL decoding algorithm with list size $L=1$ ).

\section{A. Architecture Description}

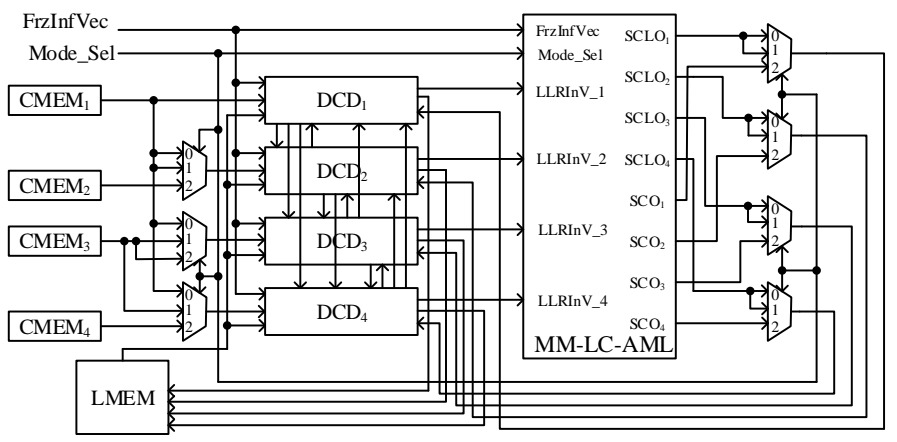

Fig. 6. Top architecture of the MM-SCL decoder when $n_{d}=4$.

Assuming $n_{d}=4$, the top architecture of the MM-SCL decoder is shown in Fig. 6. It has four blocks of channel memory, $\mathrm{CMEM}_{i}(1 \leq i \leq 4)$, to store four received codewords since the decoder of the MODE- 1 mode can deal with four received codewords simultaneously. Block $\mathrm{DCD}_{i}$ $(1 \leq i \leq 4)$ contains processing units to calculate LLRs, and partial-sum units to update partial-sum for each list. The intermediate LLRs calculated by $\mathrm{DCD}_{i}$ are stored in block LMEM. Designs for processing units, partial-sum units and the interface between processing units and LMEM adopt blocks of the reduced-latency tree-based SCL decoder in [27]. We focus on the additional logic to support multi-mode features. Mode_Sel is a two-bit control word to select the decoding mode of the MM-SCL decoder, shown in Table III.

TABLE III

CONTROL WORD FOR THE DECODING MODE OF THE MM-SCL DECODER WITH $n_{d}=4$.

\begin{tabular}{|c|c|c|}
\hline Mode_Sel & Decoding Mode & Notation \\
\hline 0 & SCL with $L=4$ & MODE-4 \\
\hline 1 & SCL with $L=2$ & MODE-2 \\
\hline 2 & SC & MODE-1 \\
\hline
\end{tabular}

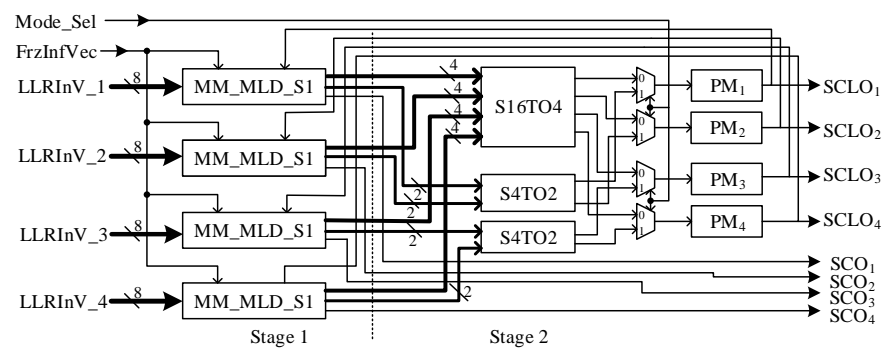

Fig. 7. Top architecture of MM-LC-AML for the MM-SCL decoder.

Block MM-LC-AML performs the LC-AML decoding function for different types of leaf nodes and is supposed to output the most reliable list candidate for the MODE- 1 mode, the two most reliable list candidates for the MODE- 2 mode, and the four most reliable list candidates for the MODE-4 mode. The architecture in Fig. 4 is for a fixed list size only. Here, we propose an MM-LC-AML unit (we take $n_{d}=4$ and $M=8$ as an example) shown in Fig. 7 to support the multi-mode features. When the mode is MODE-4, all of $\mathrm{SCLO}_{1}, \mathrm{SCLO}_{2}$, $\mathrm{SCLO}_{3}$, and $\mathrm{SCLO}_{4}$ are used to decode a received codeword. When the mode is MODE-2, SCLO 1 and $\mathrm{SCLO}_{2}$ are used for one of two received codewords; $\mathrm{SCLO}_{3}$ and $\mathrm{SCLO}_{4}$ are used for the other received codeword. When the mode is MODE-1, each of $\operatorname{SCO}_{i}(1 \leq i \leq 4)$ is used by an individual received codeword.

Block MM_MLD_S1 performs the same function as block MLD_S1_q4 in Fig. 5, except that block MM_MLD_S1 supports multiple modes. This can be accomplished by simply adding an S4 block between block S32TO4 and the adder at the bottom right of Fig. 5(a). This implementation has a disadvantage: the delay of the critical path is increased due to the extra block in the data path and the decoding latency is increased.

To avoid the unnecessary increase of the decoding latency, we redesign the MLD_S1 block for the MODE-1 and MODE2 modes, respectively, called MLD_S1_q1 and MLD_S1_q2, 


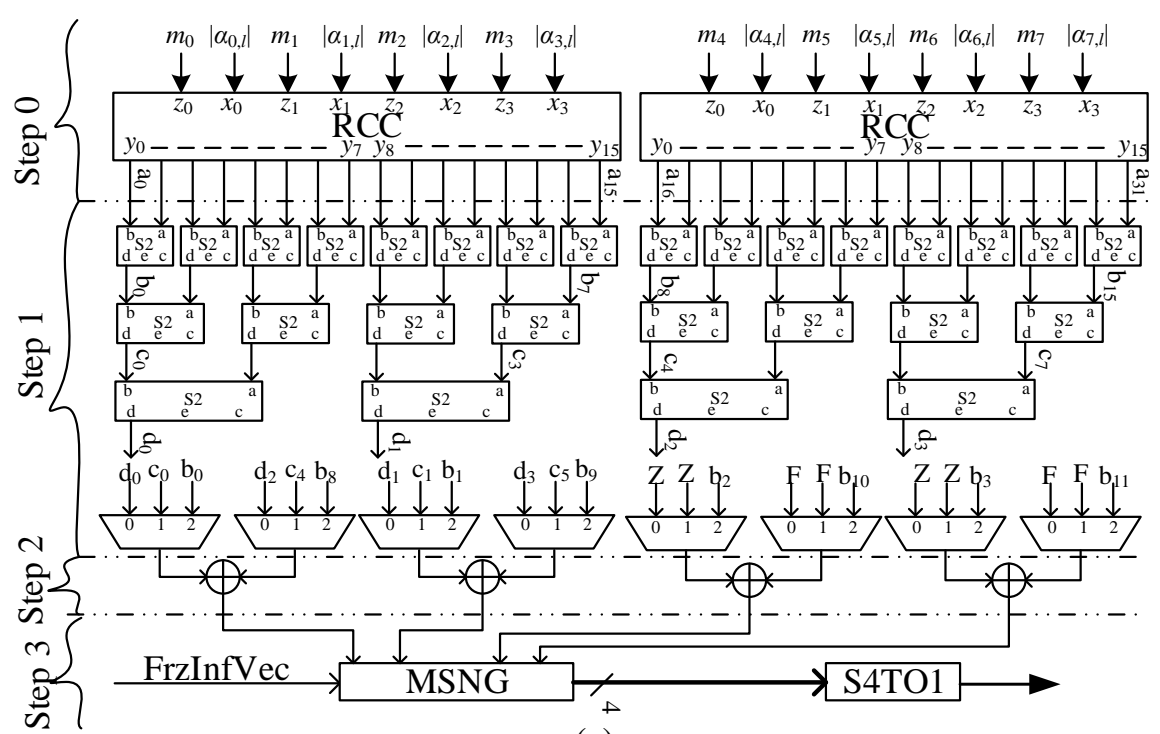

(a)



(b)

Fig. 8. (a) Design of MLD_S1_q1 for $q=1$, (b) design of MLD_S1_q2 for $q=2$.

shown in Fig. 8(a) and 8(b). Symbol values for ' $Z$ ' and ' $F$ ' are four-bit vectors ' 0000 ' and ' 1111 ', respectively. Hence, the symbol value calculated from ' $Z$ ' and ' $F$ ' is '11111111', which is guaranteed to be an invalid symbol value for our designs.

If MODE-2 is used, the control words for patterns ' $\mathcal{F} \mathcal{F} \mathcal{D D D D D D}$ ', ' $\mathcal{F D D D D D D D}$ ', and ' $\mathcal{F} \mathcal{F} \mathcal{F} \mathcal{D D D D D}$ ' are 0,1 , and 2 , respectively; for the remaining patterns, the control words are 3 . If MODE-1 is used, the control words for patterns ' $\mathcal{F} \mathcal{F D D D D D D}$ ', ' $\mathcal{F D D D D D D D}$ ', and ' $\mathcal{F} \mathcal{F} \mathcal{F} \mathcal{D D D D D}$ ' are 0,0 , and 1 , respectively; for the remaining patterns, the control words are 2.

Actually, MLD_S1_q4, MLD_S1_q2 and MLD_S1_q1 are integrated together instead of three individual blocks in block MM_MLD_S1, since they have the same circuitry for Step
0. Furthermore, sorting units of the top row of Step 1 in these three designs can also be reused because block S8TO4 contains several S4 blocks and S2 blocks. The hardware sharing reduces the additional area for supporting multiple modes and improves area efficiency without increasing the critical path delay.

Compared with the AE-SCL decoder in Section V-B, the MM-SCL decoder needs additional hardware for supporting multiple modes. The main area increase is the additional three blocks of channel memories and the hardware of MM_MLD_S1 to support the MODE-2 and MODE-1 modes.

Frame error rates of different modes for the MM-SCL decoder to decoder all the three codes are shown in Fig. 9 which shows that, regarding the frame error rates, MODE-4 $<$ MODE-2 < MODE-1. 


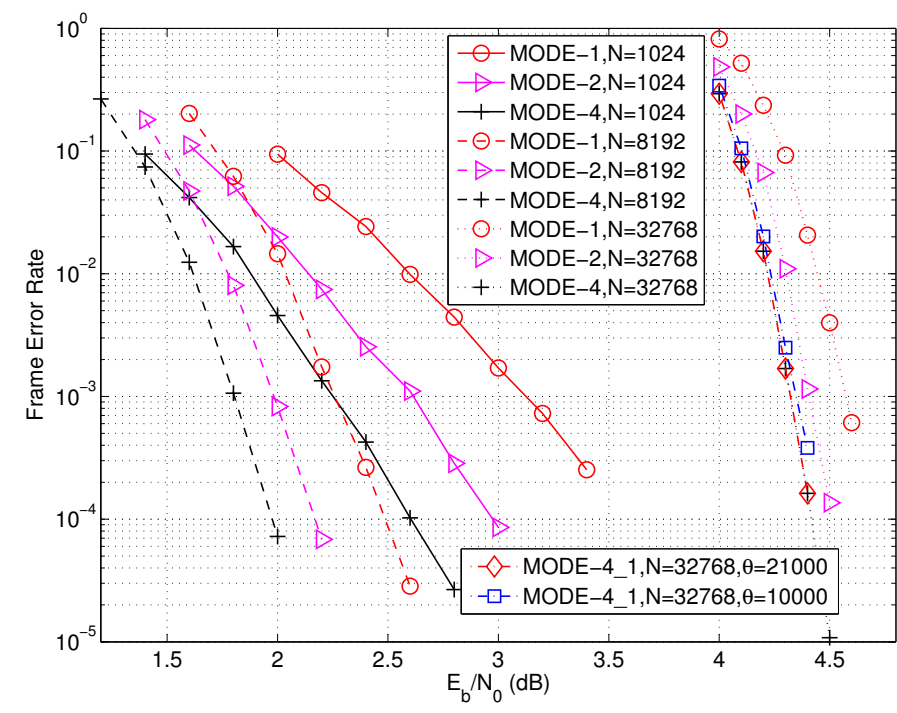

Fig. 9. Frame error rates of different modes for the MM-SCL decoder.

\section{B. Variation of modes}

An additional feature of the MM-SCL decoder is that Mode_Sel can be changed during the decoding procedure of a received word. This doe not need any additional hardware. It means that the SCL algorithm with different list sizes can be used to deal with different segments of a received word. For example, for a $(32768,29504)$ polar code, $u_{1}^{21000}$ is decoded by the SCL algorithm of $L=4$ and $u_{21001}^{32768}$ is decoded by the SC algorithm. For the first 21000 bits, the decoder is in MODE-4 and four lists are maintained. The remaining bits are decoded by the SC algorithm for each list. This mode is called MODE-4_1. By choosing the switching point $\theta$ (where the mode switches) of Mode_Sel carefully, the decoding latency can be improved slightly without any observed performance loss as shown in Fig. 9. The decoding latency of the MODE-4 mode is 6718 cycles. MODE-4_1 takes 6530 cycles to decode a received codeword and improves the throughput slightly. To reduce the decoding latency further, a smaller switching point can be used at the expense of small performance loss. If $\theta=10000$, MODE-4_1 has a decoding latency of 6206 cycles and has a performance loss of about $0.03 \mathrm{~dB}$ compared with MODE-4 as shown in Fig. 9 but still has a better performance than MODE-2. Hence, MODE-4_1 provides a more flexible way to achieve a decoding latency between those of the MODE-4 mode and the MODE-1 mode.

Therefore, the variation of modes provides a way for the MM-SCL decoder to reduce decoding latency further somewhat without noticeable performance loss and improves area efficiency further. It can also be used when decoding needs to be finished as soon as possible due to external reasons, such as buffer overflow.

\section{Synthesis Results}

The MM-SCL decoder are implemented for the aforementioned three codes. For the $(1024,512)$, the areas of the channel memory and the ML decoding unit are listed in Table IV. It shows that the increased area of the MM-SCL decoder over the AE-SCL decoder is dominated by the area of additional three blocks of channel memory. Due to the hardware sharing, the increased area of the ML decoding unit is small.

TABLE IV

AREAS $\left(\right.$ IN MM $^{2}$ ) OF THE CHANNEL MEMORY AND THE ML DECODING UNIT FOR MM-SCL AND AE-SCL DECODERS WHEN $N=1024$ AND $\mathrm{R}=0.5$.

\begin{tabular}{|c|c|c|c|}
\hline & MM-SCL & AE-SCL & Difference \\
\hline Area of Channel Memory & 0.484 & 0.121 & 0.363 \\
\hline Area of ML Decoding Unit & 0.513 & 0.456 & 0.057 \\
\hline
\end{tabular}

Synthesis results of MM-SCL decoders for different polar codes are listed in Tables $\mathrm{V}, \mathrm{VI}$ and $\mathrm{VII}$ The decoding latency of the MODE-2 mode is smaller than that of the MODE4 mode and the decoder has the smallest decoding latency with the MODE-1 mode. This is because MLD_S1_q2 and MLD_S1_q1 have shorter data paths. Therefore, in block MMLC-AML, three stages and two stages of pipeline registers are used by the circuitry for the MODE- 2 mode and the MODE1 mode, respectively. If MODE-4_1 is used, the MM-SCL decoder can achieve a smaller latency than the MODE-4 mode and the MODE-2 mode.

Compared with the AE-SCL decoder, the MM-SCL decoder in the MODE-4 mode has a smaller area efficiency due to the additional circuitry for supporting multiple modes. However, the MM-SCL decoder is more flexible to provide multiple choices of output throughput and decoding latency, which is more suitable for variable communication channels and applications. If a higher throughput or a smaller decoding latency is required, the MM-SCL decoder can be switched to the MODE-2, MODE-1 or MODE-4_1 mode.

Compared with the decoder in [27], for $N=1024$ and $N=8192$, the MM-SCL decoder has a smaller area and a better area efficiency. For $N=32768$, the area of the MMSCL decoder is bigger than that of the decoder in [27] because the additional circuitry to support multiple modes is larger than the saving due to the low-complexity ML decoding unit in the MM-SCL decoder. For the $(1024,512)$ code, under the MODE-4, MODE-2, and MODE-1 modes, the MM-SCL decoder provides area-efficiencies of 1.59, 3.51, and 8.39 times of area-efficiency of the decoder in [27], respectively.

Compared with decoders in [10], [24], [25], the advantage in area efficiency of the MM-SCL decoder is more significant. This advantage comes from two aspects. The first is that the tree-based low-latency SCL architecture in [27] is adopted for the MM-SCL decoder. This helps to reduce the decoding latency. The second is due to the low-complexity AML decoding unit. For $N=1024$, the MM-SCL decoder in MODE-4 mode provides an area efficiency of 2.72, 6.77, and 2.60 times of area efficiencies of SCL decoders in [10], [24], [25], respectively. When the mode is MODE-1, the ratios of the area efficiency of the MM-SCL decoder over those of SCL decoders in [10], [24], [25] are 14.37, 35.71, and 13.73, respectively.

For $N=32768$, decoding latencies and throughputs respect to different switching points of MODE-4_1 are also provided. A smaller switching point leads to a smaller latency. When the switching point is 10000 , the latency of MODE-4_1 is 
TABLE V

SYNTHESIZING RESULTS FOR DIFFERENT DECODERS WHEN $N=1024$ AND R $=0.5$.

\begin{tabular}{|c|c|c|c|c|c|c|c|c|c|}
\hline Decoder & AE-SCL & MODE-4 & MODE-2 & MODE-1 & $\mid 27$ & $\mid 25$ & {$[10]$} & \multicolumn{2}{|c|}{24} \\
\hline List Size & 4 & 4 & 2 & 1 & \multicolumn{5}{|c|}{4} \\
\hline Area $\left(\mathrm{mm}^{2}\right)$ & 1.89 & \multicolumn{3}{|c|}{2.31} & 3.83 & 1.70 & 1.78 & 2.14 & $4.10^{*}$ \\
\hline Clock Rate (MHz) & 409 & \multicolumn{3}{|c|}{409} & 403 & 500 & 794 & 400 & $289^{*}$ \\
\hline \# of Decoding Cycles & 391 & 391 & 357 & 304 & 371 & 1540 & 2649 & \multicolumn{2}{|c|}{1022} \\
\hline Latency (us) & 0.96 & 0.96 & 0.87 & 0.74 & 0.92 & 3.08 & 3.34 & 2.56 & $3.54^{*}$ \\
\hline NIT (Mbps) & 547 & 547 & 1208 & 2887 & 570 & 155 & 154 & 200 & $144^{*}$ \\
\hline Area Eff. $\left(\mathrm{Mbps} / \mathrm{mm}^{2}\right)$ & 289 & 237 & 523 & 1250 & 149 & 91 & 87 & 93 & $35^{*}$ \\
\hline
\end{tabular}

* Original synthesis results in [24] are based on an ST 65nm CMOS technology. For a fair comparison, synthesis results scaled to a $90 \mathrm{~nm}$ technology are used in the comparison.

TABLE VI

SYNTHESIZING RESULTS FOR DIFFERENT DECODERS WHEN $N=8192$ AND R=0.5.

\begin{tabular}{|c|c|c|c|c|c|c|c|}
\hline Decoder & AE-SCL & MODE-4 & MODE-2 & MODE-1 & 27 & $\left.25\right|^{\ddagger}$ & $10]^{\dagger}$ \\
\hline List Size & 4 & 4 & 2 & 1 & & 4 & \\
\hline Area $\left(\mathrm{mm}^{2}\right)$ & 4.49 & \multicolumn{3}{|c|}{5.51} & 6.46 & 7.32 & 12.73 \\
\hline Clock Rate (MHz) & 398 & \multicolumn{3}{|c|}{398} & 398 & 434 & 794 \\
\hline \# of Decoding Cycles & 2542 & $\overline{2542}$ & 2323 & 1975 & 2367 & 11700 & 20736 \\
\hline Latency (us) & 6.39 & 6.39 & 5.84 & 4.96 & 5.95 & 26.96 & 26.12 \\
\hline NIT (Mbps) & 670 & 670 & 1473 & 3503 & 723 & 150 & 156 \\
\hline Area Eff. $\left(\mathrm{Mbps} / \mathrm{mm}^{2}\right)$ & 149 & 122 & 267 & 636 & 112 & 20 & 12 \\
\hline
\end{tabular}

¥ The decoder architecture in [25] has been re-synthesized with the TSMC 90nm CMOS technology.

$\dagger$ These results for the decoder in [10] are estimated conservatively.

TABLE VII

SYNTHESIZING RESULTS FOR DIFFERENT DECODERS WHEN N=32768 AND R=0.9.

\begin{tabular}{|c|c|c|c|c|c|c|c|c|c|}
\hline Decoder & AE-SCL & MODE-4 & \multicolumn{2}{|c|}{ MODE-4_1 } & MODE-2 & MODE-1 & 27 & {$[25]^{\mp}$} & {$[10]^{\dagger}$} \\
\hline List Size & 4 & 4 & $4 *$ & $4^{\star}$ & 2 & 1 & & 4 & \\
\hline Area $\left(\mathrm{mm}^{2}\right)$ & 9.97 & \multicolumn{5}{|c|}{11.93} & 11.89 & 15.8 & 50.41 \\
\hline Clock Rate $(\mathrm{MHz})$ & 350 & \multicolumn{5}{|c|}{350} & 359 & 389 & 794 \\
\hline \# of Decoding Cycles & 6718 & 6718 & 6530 & 6206 & 6300 & 5368 & 6492 & 65813 & 96576 \\
\hline Latency (us) & 19.19 & 19.19 & 18.66 & 17.73 & 18 & 15.34 & 18.08 & 169.19 & 121.63 \\
\hline NIT (Mbps) & 1662 & 1662 & 1714 & 1811 & 3564 & 8499 & 1772 & 165 & 242 \\
\hline Area Eff. $\left(\mathrm{Mbps} / \mathrm{mm}^{2}\right)$ & 167 & 139 & 144 & 152 & 299 & 712 & 149 & 10 & 5 \\
\hline
\end{tabular}

$¥$ The decoder architecture in [25] has been re-synthesized with the TSMC 90nm CMOS technology.

$\dagger$ These results for the decoder in $[10]$ are estimated conservatively.

* The switching point for variation of modes is 21000 .

* The switching point for variation of modes is 10000 .

even smaller than that of MODE-2. Compared with MODE-4, improvements on throughput and latency are about $8 \%$.

\section{CONCLUSION}

In this paper, the divide-and-conquer method is applied to SC-based algorithms in the probability domain. By extending this idea, a divide-and-conquer AML decoding unit for SCLbased polar decoder is proposed. By examining frozen-location patterns of polar codes, an efficient hardware design for a simplified divide-and-conquer AML decoding unit is developed. To adapt to different throughput and latency requirements, the MM-SCL polar decoder is proposed in this paper. Synthesis results show that our implementations for our MM-SCL decoder and SCL decoder with the LC-AML unit achieve better area efficiencies than existing SCL polar decoders.

\section{APPENDIX}

\section{Proof of Proposition 1 .}

(a) First, $0<z_{1,1}=2 \epsilon-\epsilon^{2}=1-(1-\epsilon)^{2}<1$. Second, $0<z_{1,2}=\epsilon^{2}<1$. Then, by induction, for $i \geq 1$ and $1 \leq j \leq 2^{i}, 0<z_{i, j}<1$ is satisfied. (b) For any $i \geq 1$ and $1 \leq j \leq 2^{i}, z_{i, 2 j-1}-z_{i, 2 j}=2 z_{i-1, j}-$ $z_{i-1, j}^{2}-z_{i-1, j}^{2}=2 z_{i-1, j}\left(1-z_{i-1, j}\right)$. By Proposition 1 (a), $z_{i, 2 j-1}-z_{i, 2 j}>0 \Rightarrow z_{i, 2 j-1}>z_{i, 2 j}$.

(c) By Proposition 11(b), $z_{i, 4 j-3}>z_{i, 4 j-2}$ and $z_{i, 4 j-1}>$ $z_{i, 4 j} . z_{i, 4 j-2}-z_{i, 4 j-1}=2 z_{i-2, j}^{2}\left(1-z_{i-2, j}\right)^{2}$. By Proposition 11.a), $z_{i, 4 j-2}-z_{i, 4 j-1}>0 \Rightarrow z_{i, 4 j-2}>z_{i, 4 j-1}$.

Therefore, $z_{i, 4 j-3}>z_{i, 4 j-2}>z_{i, 4 j-1}>z_{i, 4 j}$.

(d) By Proposition 11(c), $z_{i, 8 j-7}>z_{i, 8 j-6}>z_{i, 8 j-5}>$ $z_{i, 8 j-4}$ and $z_{i, 8 j-3}>z_{i, 8 j-2}>z_{i, 8 j-1}>z_{i, 8 j}$. We also have $z_{i, 8 j-5}>z_{i, 8 j-3}$ and $z_{i, 8 j-4}>z_{i, 8 j-2}$ because $z_{i, 4 j-2}>z_{i, 4 j-1}$.

Now let us compare $z_{i, 8 j-4}$ and $z_{i, 8 j-3}$,

$$
\begin{aligned}
& z_{i, 8 j-4}-z_{i, 8 j-3}=-2 z_{i-3, j}^{2}\left(1-z_{i-3, j}\right)^{2} \\
& \times\left(2+4 z_{i-3, j}-5 z_{i-3, j}^{2}+2 z_{i-3, j}^{3}-z_{i-3, j}^{4}\right) .
\end{aligned}
$$

By Proposition 11(a), $z_{i, 8 j-4}<z_{i, 8 j-3}$.

Therefore, $z_{i, 8 j-7}>z_{i, 8 j-6}>z_{i, 8 j-5}>z_{i, 8 j-3}>$ $z_{i, 8 j-4}>z_{i, 8 j-2}>z_{i, 8 j-1}>z_{i, 8 j}$.

\section{REFERENCES}

[1] E. Arıkan, "Channel polarization: A method for constructing capacityachieving codes for symmetric binary-input memoryless channels," IEEE 
Transactions on Information Theory, vol. 55, no. 7, pp. 3051-3073, July 2009.

[2] E. Sasoglu, I. Telatar, and E. Arıkan, "Polarization for arbitrary discrete memoryless channels," in Proceedings of IEEE Information Theory Workshop, Oct 2009, pp. 144-148.

[3] I. Tal and A. Vardy, "List decoding of polar codes," IEEE Transactions on Information Theory, vol. 61, no. 5, pp. 2213-2226, May 2015.

[4] E. Arıkan, "Systematic polar coding," IEEE Communications Letters, vol. 15 , no. 8 , pp. 860-862, August 2011.

[5] K. Niu and K. Chen, "CRC-aided decoding of polar codes," IEEE Communications Letters, vol. 16, no. 10, pp. 1668-1671, October 2012.

[6] S. Kahraman and M. Celebi, "Code based efficient maximum-likelihood decoding of short polar codes," in Proceedings of IEEE International Symposium on Information Theory, July 2012, pp. 1967-1971.

[7] K. Niu, K. Chen, and J. Lin, "Low-complexity sphere decoding of polar codes based on optimum path metric," IEEE Communications Letters, vol. 18, no. 2, pp. 332-335, February 2014.

[8] E. Arıkan, H. Kim, G. Markarian, U. Ozgur, and E. Poyraz, "Performance of short polar codes under $\mathrm{ml}$ decoding," in Proceedings of ICT Mobile Summit Conference, 2009.

[9] Y. S. Park, Y. Tao, S. Sun, and Z. Zhang, "A 4.68gb/s belief propagation polar decoder with bit-splitting register file," in Symposium on VLSI Circuits Digest of Technical Papers, June 2014, pp. 1-2.

[10] A. Balatsoukas-Stimming, M. Bastani Parizi, and A. Burg, "LLR-based successive cancellation list decoding of polar codes," IEEE Transactions on Signal Processing, vol. 63, no. 19, pp. 5165-5179, Oct 2015.

[11] J. Lin and Z. Yan, "An efficient list decoder architecture for polar codes," IEEE Transactions on Very Large Scale Integration (VLSI) Systems, 2015, accepted and to appear, available on IEEE Explore, DOI: 10.1109/TVLSI.2014.2378992.

[12] C. Leroux, A. Raymond, G. Sarkis, and W. Gross, "A semi-parallel successive-cancellation decoder for polar codes," IEEE Transactions on Signal Processing, vol. 61, no. 2, pp. 289-299, Jan 2013.

[13] G. Sarkis, P. Giard, A. Vardy, C. Thibeault, and W. Gross, "Fast polar decoders: Algorithm and implementation," IEEE Journal on Selected Areas in Communications, vol. 32, no. 5, pp. 946-957, May 2014.

[14] A. Balatsoukas-Stimming, A. Raymond, W. Gross, and A. Burg, "Hardware architecture for list successive cancellation decoding of polar codes," IEEE Transactions on Circuits and Systems II: Express Briefs, vol. 61, no. 8, pp. 609-613, Aug 2014.

[15] G. Sarkis and W. Gross, "Increasing the throughput of polar decoders," IEEE Communications Letters, vol. 17, no. 4, pp. 725-728, Apr. 2013.

[16] C. Zhang and K. Parhi, "Latency analysis and architecture design of simplified sc polar decoders," IEEE Transactions on Circuits and Systems II: Express Briefs, vol. 61, no. 2, pp. 115-119, Feb. 2014.

[17] _ - "Low-latency sequential and overlapped architectures for successive cancellation polar decoder," IEEE Transactions on Signal Processing, vol. 61, no. 10, pp. 2429-2441, May 2013.

[18] A. Raymond and W. Gross, "A scalable successive-cancellation decoder for polar codes," IEEE Transactions on Signal Processing, vol. 62 , no. 20, pp. 5339-5347, Oct. 2014.

[19] C. Xiong, J. Lin, and Z. Yan, "Symbol-based successive cancellation list decoder for polar codes," in Proceedings of IEEE Workshop on Signal Processing Systems, Belfast, UK, October 2014, pp. 198-203.

[20] G. Sarkis, P. Giard, A. Vardy, C. Thibeault, and W. Gross, "Increasing the speed of polar list decoders," in 2014 IEEE Workshop on Signal Processing Systems (SiPS), Oct 2014, pp. 1-6.

[21] I. Tal and A. Vardy, "List decoding of polar codes," in Proceedings of IEEE International Symposium on Information Theory, July 2011, pp. $1-5$.

[22] B. Li, H. Shen, and D. Tse, "Parallel decoders of polar codes," arXiv:1309.1026, Sep. 2013. [Online]. Available: http://arxiv.org/abs/ 1309.1026

[23] B. Li, H. Shen, D. Tse, and W. Tong, "Low-latency polar codes via hybrid decoding," in Proceedings of 2014 8th International Symposium on Turbo Codes and Iterative Information Processing, Aug. 2014, pp. 223-227.

[24] B. Yuan and K. Parhi, "Low-latency successive-cancellation list decoders for polar codes with multibit decision," IEEE Transactions on Very Large Scale Integration (VLSI) Systems, 2014, accepted and to appear, available on IEEE Explore, DOI: 10.1109/TVLSI.2014.2359793.

[25] C. Xiong, J. Lin, and Z. Yan, "Symbol-decision successive cancellation list decoder for polar codes," IEEE Transactions on Signal Processing, 2015, accepted and to appear, available on IEEE Explore, DOI: 10.1109/TSP.2015.2486750.
[26] J. Lin, C. Xiong, and Z. Yan, "A reduced latency list decoding algorithm for polar codes," in Proceedings of IEEE Workshop on Signal Processing Systems (SiPS 2014), Belfast, UK, October 2014, pp. 56-61. [Online]. Available: http://arxiv.org/abs/1405.4819

[27] —, "A high throughput list decoder architecture for polar codes," arXiv:1510.02574, Oct. 2015. [Online]. Available: http: //arxiv.org/abs/1510.02574

[28] E. Arikan, "A performance comparison of polar codes and Reed-Muller codes," IEEE Communications Letters, vol. 12, no. 6, pp. 447-449, June 2008.

[29] D. Wu, Y. Li, and Y. Sun, "Construction and block error rate analysis of polar codes over awgn channel based on gaussian approximation," IEEE Communications Letters, vol. 18, no. 7, pp. 1099-1102, July 2014.

[30] J. Lin and Z. Yan, "Efficient list decoder architecture for polar codes," in Proceedings of IEEE International Symposium on Circuits and Systems, June 2014, pp. 1022-1025.

[31] K. E. Batcher, "Sorting networks and their applications," in AFIPS Proceeding of the Spring Joint Computer Conference, 1968, pp. 307314. 